\title{
Robust Audio Event Recognition with 1-Max Pooling Convolutional Neural Networks
}

\author{
Huy Phan ${ }^{\star \dagger}$, Lars Hertel ${ }^{\star}$, Marco Maass ${ }^{\star}$, and Alfred Mertins ${ }^{\star}$ \\ ${ }^{\star}$ Institute for Signal Processing, University of Lübeck \\ ${ }^{\dagger}$ Graduate School for Computing in Medicine and Life Sciences, University of Lübeck \\ $\{$ phan, hertel, maass, mertins\}eisip.uni-luebeck. de
}

\begin{abstract}
We present in this paper a simple, yet efficient convolutional neural network $(\mathrm{CNN})$ architecture for robust audio event recognition. Opposing to deep CNN architectures with multiple convolutional and pooling layers topped up with multiple fully connected layers, the proposed network consists of only three layers: convolutional, pooling, and softmax layer. Two further features distinguish it from the deep architectures that have been proposed for the task: varying-size convolutional filters at the convolutional layer and 1-max pooling scheme at the pooling layer. In intuition, the network tends to select the most discriminative features from the whole audio signals for recognition. Our proposed CNN not only shows state-of-the-art performance on the standard task of robust audio event recognition but also outperforms other deep architectures up to $4.5 \%$ in terms of recognition accuracy, which is equivalent to $76.3 \%$ relative error reduction.
\end{abstract}

Index Terms: audio event recognition, robustness, convolutional neural networks, 1-max pooling

\section{Introduction}

The success of deep architectures in many applications is explained by their ability to discover multiple levels of features from data. Inspired by this, many deep neural networks have recently been proposed for audio event recognition. In [1, 2], deep neural networks (DNNs) are first initialized using unsupervised training with deep belief networks (DBNs) [3] and then trained by the standard backpropagation. In order to deal with event overlap, DNNs with multi-label classification schemes have also been proposed [4]. Recently, various deep CNN architectures with multiple convolutional and pooling layers for hierarchical feature extraction have also been employed $[5,6,7,8]$. Although these deep networks showed promising performance, especially under difficult conditions such as under interference $[1,6]$ and event overlapping [4], they come with a significant shortcoming. These deep architectures require equal-size inputs while the nature of audio events exhibits high intra- and inter-class temporal durations. To go around this issue, the signals were decomposed into equal segments and the models were then trained on these local features. In turn, the evaluation also took place on local features followed by some voting schemes, e.g. majority voting $[1,7,8]$ and probability voting [7], to obtain a global classification label. Although this adaptation helps to facilitate the training and testing of the models, it is incapable of capturing the shift-invariance property [9] that the cochlea and auditory nerve in the auditory system have [10]. This is really undesirable since a particular feature could be replicated at any time in the signal instead of its local segments.
We present a convolutional neural network architecture for robust audio event recognition that is able to address these issues. Our architecture is much simpler and more "shallow". It consists of three layers: convolutional, pooling, and softmax layer. The convolution layer coupled with the pooling layer are responsible for feature extraction and the final softmax layer is in charge of classification. Our proposed architecture is different from the deep ones that have been used for the task in many aspects. Foremost, it takes the whole signals of audio events as input instead of their small fractions. Second, we do not fix the size of the convolutional filters at the convolutional layer as in conventional CNNs but allow multiple filters with different sizes to be learned simultaneously. Consequently, we are able to capture features at multiple resolutions of audio signals. Third, we do not pursuit subsampling at the pooling layer but 1-max pooling scheme. As a result, with the feature map induced by convolving one of the filters on an input signal, we only select the most prominent feature. The prominent features produced by all filters are finally concatenated and presented to the final softmax layer for classification. Furthermore, owing to the 1-max pooling, the inputs to the network can be of any arbitrary size. That is, we can naturally deal with the intra- and inter-class temporal variation of audio events. Lastly, each convolutional filter can be thought of playing the role of a cochlear filter which spikes on a specific feature of the signal $[11,10]$. In addition, the feature is allowed to happen at any time in the signal, i.e. it is shift-invariant.

\section{The proposed approach}

In this section we will present the spectrogram image features that are used to represent audio signals. Afterwards, our proposed $\mathrm{CNN}$ architecture will be described. The spectrogram images are used as inputs for the network.

\subsection{Spectrogram image features (SIF)}

Given an audio signal, it is decomposed into overlapping segments from which a spectrogram is generated by short-time Fourier transform. The short-time spectral column representing a length- $L$ segment $s_{t}(n)$ at the time index $t$ is given by

$$
\mathbf{S}(f, t)=\left|\sum_{n=0}^{L-1} s_{t}(n) \phi(n) e^{\frac{-j 2 \pi n f}{L}}\right|,
$$

where $f=0, \ldots,\left(\frac{L}{2}-1\right)$ and $\phi(n)$ denotes a $L$-point Hamming window. The spectrogram is then down-sampled in frequency to keep a $F$-bin frequency resolution by averaging over a window of length $W=\lfloor L / 2 F\rfloor$. 
A de-noising step is finally performed by subtracting the minimum value from each spectral vector over time:

$$
\mathbf{S}_{d n}(f, t)=\mathbf{S}(f, t)-\min _{t}(\mathbf{S}(f, t)),
$$

for $f=0, \ldots,(F-1)$. The short-time energy $e(t)$ can also be appended to the spectrogram image as an augmented feature:

$$
e(t)=\sum_{f=0}^{F-1} \mathbf{S}_{d n}(f, t) .
$$

Our proposed SIF features are similar to those in [1]. However, instead of classifying on equal spectro-temporal patches of the images, our classification is efficiently performed on the whole varying-size spectrogram images.

\subsection{1-Max Pooling CNN}

The proposed network consists of three layers, including convolutional, pooling, and softmax layer as illustrated in Figure 1.

\subsubsection{Convolutional layer}

We aim to use the convolutional layer to extract discriminative features within the whole signals that are useful for the classification task at hand. Suppose that a spectrogram image presented to the network is given in the form of a matrix $\mathbf{S} \in \mathbb{R}^{F \times T}$ where $F$ and $T$ denote the number of frequency bins and the number of audio segments, respectively. We then perform convolution on it via linear filters. For simplicity, we only consider convolution in time direction, i.e. we fix the height of the filter to be equal to the number of frequency bins $F$ and vary the width of the filter to cover different number of adjacent audio segments.

Let us denote a filter by the weight vector $\mathbf{w} \in \mathbb{R}^{F \times w}$ with the width $w$. Therefore, the filter contains $F \times w$ parameters that need to be learned. We further denote the adjacent spectral columns (e.g. audio segments) from $i$ to $j$ by $\mathbf{S}[i: j]$. The convolution operation $*$ between $\mathbf{S}$ and $\mathbf{w}$ results in the output vector $\mathbf{O}=\left(o_{1}, \ldots, o_{T-w+1}\right)$ where

$$
o_{i}=(\mathbf{S} * \mathbf{w})_{i}=\sum_{k, l}(\mathbf{S}[i: i+w-1] \odot \mathbf{w})_{k, l} .
$$

Here, $\odot$ denotes the element-wise multiplication. We then apply an activation function $h$ to each $o_{i}$ to induce the feature map $\mathbf{A}=\left(a_{1}, \ldots, a_{T-w+1}\right)$ for this filter:

$$
a_{i}=h\left(o_{i}+b\right)
$$

where $b \in \mathbb{R}$ is a bias term. Among the common activation fuctions, we chose Rectified Linear Units (ReLU) [12] due to their computational efficiency:

$$
h(x)=\max (0, x) .
$$

To allow the network to extract complementary features and enrich the representation, we learn $P$ different filters simultaneously. Furthermore, the use of multiple resolution levels has been shown important for the task [5] as the time duration that yields salient features may vary depending on the event categories. In order to account for this, we learn $Q$ different sets of $P$ filters, each of which has different width $w$ to form totally $Q \times P$ filters.

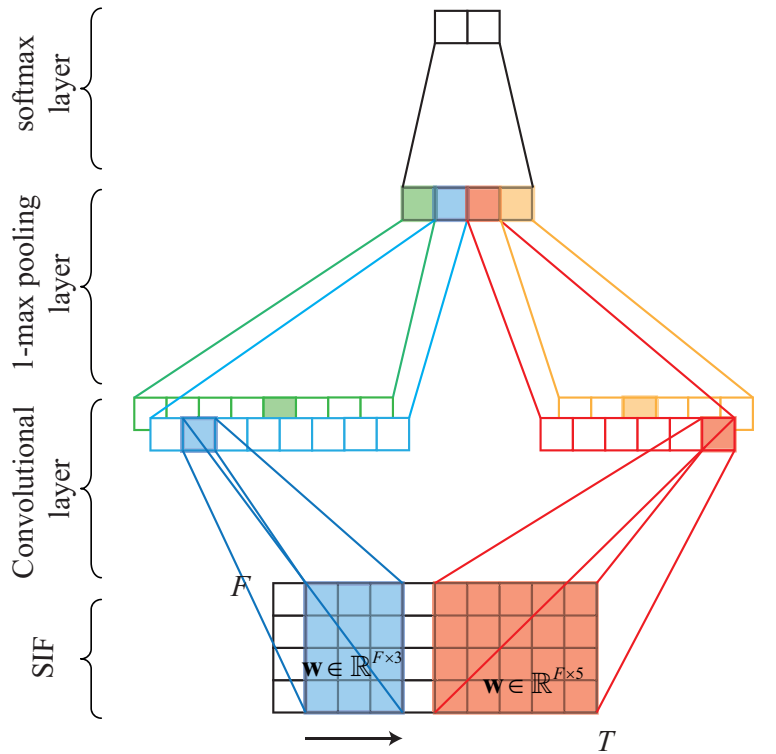

Figure 1: Illustration of 1-max pooling CNN architecture. The network consists of two filter sets with two different widths $w=\{3,5\}$ at the convolutional layer. There are two individual filters on each filter set.

\subsubsection{1-max pooling layer}

The feature maps produced by the convolution layer are forwarded to the pooling layer. We employ 1-max pooling function [13] on a feature map to reduce it to a single most dominant feature. Pooling on $Q \times P$ feature maps results in $Q \times P$ features that will be joined to form a feature vector inputted to the final softmax layer. This pooling strategy offers a unique advantage. That is, although the dimensionality of the feature maps varies depending on the length of audio events and the width of the filters, the pooled feature vectors have the same size of $P \times Q$. The same strategy has recently been proved useful in different tasks of natural language processing owing to its ability to cope with varying-size input texts, such as sentences $[14,15]$. Coupled with the 1-max pooling function, each filter in the convolutional layer is optimized to detect a specific feature that is allowed to occur at any time in a signal.

\subsubsection{Softmax layer}

The fixed-size feature vector after the pooling layer is subsequently presented to the standard softmax layer to compute the predicted probability over the class labels. The network is trained by minimizing the cross-entropy error. This is equivalent to minimizing the KL-divergence between the prediction distribution $\hat{y}$ and the target distribution $y$. With the binary onehot coding scheme and the network parameter $\theta$, the error for $N$ training samples is given by:

$$
E(\theta)=-\frac{1}{N} \sum_{i=1}^{N} y_{i} \log \left(\hat{y}_{i}(\theta)\right)+\frac{\lambda}{2}\|\theta\|^{2} .
$$

The hyper-parameter $\lambda$ governs the trade-off between the error term and the $\ell_{2}$-norm regularization term. For regularization purposes, we also employ dropouts [16] at this layer by randomly setting values in the weight vector to zero with a predefined probability. The optimization is performed using the Adam gradient descent algorithm [17]. 


\section{Experiments}

\subsection{Databases}

We set up the standard experiment of the robust audio event recognition task similar to current state-of-the-art works $[18,1$, $6]$ so that the results are comparable.

Audio event database. We targeted 50 sound event categories ${ }^{1}$ from the Real Word Computing Partnership (RWCP) Sound Scene Database in Real Acoustic Environments [19]. For each category, we randomly selected 80 sound instances which were divided into 50 instances for training and 30 instances for testing. Out of 50 training instances, we left out 10 instances for validation, and other 40 instances were used to tune the networks. It turns out that there are totally 2000,500 , and 1500 event instances for training, validation, and testing purpose, respectively.

Noise database. As in $[18,1,6]$, we chose four different environmental noises from NOISEX-92 database [20], including "Destroyer Control Room", "Speech Bable", "Factory Floor 1", and "Jet Cockpit 1". Beside clean signals, we also created noise-corrupted signals by randomly choosing one of four noise signals to add to the clean signals at random starting points. The noise signals were added with different level of 20,10 , and 0 $\mathrm{dB}$ signal-to-noise ratio (SNR). We evaluate both mismatched condition (tranining with only clean event instances) and multicondition (training with both clean and noise-corrupted event instances).

\subsection{Parameters}

Audio signals sampled at $16 \mathrm{kHz}$ sampling frequency were divided into $100 \mathrm{~ms}$ frames with a hop of $10 \mathrm{~ms}$. Each frame was analyzed with 2048-point FFT to obtain a spectral column which is then down-sampled as described in Section 2.1 to keep $F=52$ frequency bins. Although the SIFs can be of arbitrary sizes, we zero-padded them column-wise in time direction to ease the implementation.

The proposed $\mathrm{CNN}$ architecture involves different hyperparameters which are specified in Table 1. Although the hyperparameters were set to very common values, parameter search can be done to further enhance the performance. The networks were trained using the training set for 1000 epochs (mismatched condition) and 500 epochs (multi-condition) with a minibatch size of 100. During training the networks that maximize the classification accuracy on the validation set will be retained.

\subsection{Classification systems}

We trained four different networks using our proposed architecture:

- 1MaxCNN: our proposed SIF and 1-max pooling CNN (mismatched condition).

- 1MaxCNN-E: our proposed energy-augmented SIF and 1max pooling $\mathrm{CNN}$ (mismatched condition).

- 1MaxCNN-MC: our proposed SIF and 1-max pooling CNN (multi-condition).

- 1MaxCNN-E-MC: our proposed energy-augmented SIF and 1-max pooling CNN (multi-condition).

We compare the classification accuracy against other systems $[18,1,6]$ with the standard experimental setup. They include

- MFCC-HMM [18]: Mel Frequency Cepstral Coefficients (MFCC) with a Hidden Markov Models (HMM) backend.

\footnotetext{
${ }^{1}$ The specific event categories are based on unofficial communication with Jonathan W. Dennis, the author of [18].
}

Table 1: Hyper-parameters of the proposed CNN networks.

\begin{tabular}{|l|c|}
\hline Hyper-parameter & Value \\
\hline \hline Filter sizes & $\{1,3, \ldots, 25\}$ \\
Number of filter $P$ for each size & 100 \\
Learning rate for the Adam optimizer & 0.0001 \\
Dropout rate & 0.5 \\
Regularization parameter $\lambda$ & 0.0001 \\
\hline
\end{tabular}

- MFCC-SVM [18]: MFCC with a Support Vector Machine (SVM) backend.

- ETSI-AFE [18]: above MFCC-SVM that is further evaluated with an ETSI Advanced Front End toolkit enhancement [21].

- MPEG-7 [18]: a set of 57 low-level features coupled with Principle Component Analysis (PCA) feature selection and a HMM classifier.

- Gabor [18]: Gabor features followed by single-layer perceptron feature selection and HMM classification.

- GTCC [18]: Gammatone cepstral coefficients features with a HMM backend.

- MP+MFCC [18]: MFCCs and Gabor features from top five Gabor bases found by the matching pursuit (MP) algorithm [22] backed with a HMM classifier.

- Dennis SIF [18]: a similar SIF and a SVM classifier.

- SIF-DNN [1]: a similar SIF and DNN classification (mismatched condition).

- SIF-DNN-MC [1]: a similar SIF and DNN classification (multi-condition).

- SIF-CNN [6]: a similar SIF and deep CNN classification.

- SIF-IS-CNN [6]: an enhanced SIF by smoothing and deep CNN classification.

- SIF-IS-DNN [6]: an enhanced SIF by smoothing and DNN classification.

- MelFb-CNN [6]: an enhanced SIF features with Melfilterbank analysis and deep CNN classification.

\subsection{Experimental results}

\subsubsection{Performance as a function of the filter width}

We show in Figure 2 the performance of our systems in terms of classification accuracy as a function of the filter width $w$ in different noise conditions. When $w$ varies from small to large values, the features learned by the networks are expected to change from detail to higher abstracted ones. As can be seen, in most of the cases the accuracies grow with the increase of $w$.

For the 1MaxCNN system with mismatched condition, although it shows good robustness in low to mid-range noise conditions, it is less robust in harsh noise condition of $0 \mathrm{~dB}$. In addition, when augmented with the short-time energy feature, the system 1MaxCNN-E exhibits strong sensitivity in noise conditions. However, when being trained with multi-condition data, both 1MaxCNN-MC and 1MaxCNN-E-MC expose remarkably strong robustness to all noise conditions. The reason is that presenting the networks with mutli-condition data is not only about data augmentation but also enforces them to learn noise-robust filters.

\subsubsection{Performance comparison}

The comparison on classification accuracy of our systems and the competitive systems is given in Table 2. Note that although 

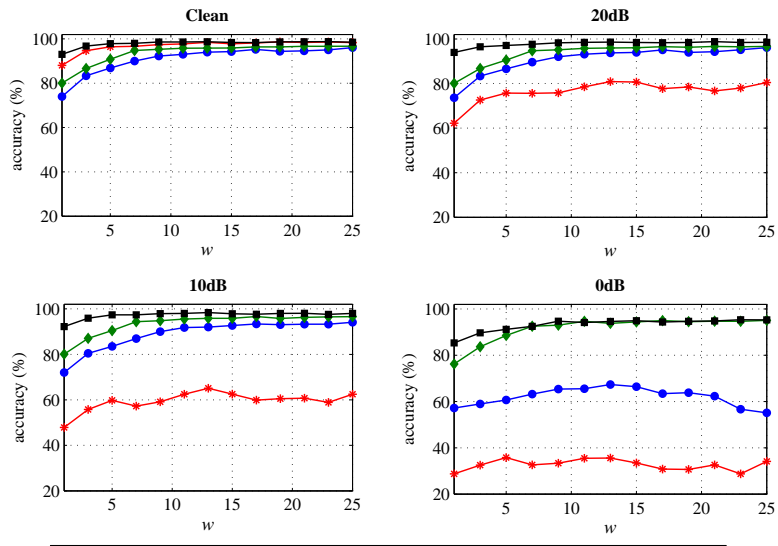

-1 MaxCNN $\rightarrow-1$ MaxCNN-E $\multimap-1$ MaxCNN-MC $\rightarrow-1$ MaxCNN-E-MC

Figure 2: Classification accuracy as a function of the filter width $w$ for different noise conditions.

our systems with a single filter size trained with multi-condition data can easily outperform the best competitor, we use the systems with multiple filter widths in $\{1,3, \ldots, 25\}$ (equivalent to $\{100,120, \ldots, 340\}$ ms respectively) for comparison here. It is partly because of the clarity's sake and partly because these systems are able to capture features on multiple resolutions and offer even better performance.

It can be seen that our system 1MaxCNN performs significantly better than all deep-architecture opponents on clean, $20 \mathrm{~dB}$, and $10 \mathrm{~dB}$ conditions although it is incomparable with the low-level feature systems (e.g. Gabor, GTCC) on the clean conditions and less robust than some deep architectures (e.g. SIF-CNN, SIF-DNN) in worst noise condition of $0 \mathrm{~dB}$. Again, when augmented with short-time energy features, the system 1MaxCNN-E exhibits its sensitivity in noise conditions although $1.1 \%$ absolute improvement can be seen in the noisefree condition.

On the other hand, our multi-condition trained systems 1MaxCNN-MC and 1MaxCNN-E-MC show superior performance compared to all deep-architecture opponents in all testing conditions, especially in the hardest one of $0 \mathrm{~dB}$. Compared to the best deep-architecture competitor (i.e. SIF-IS-CNN), 1MaxCNN-MC shows absolute gains of $1.1 \%, 1.0 \%, 2 \%$, and $12.2 \%$ on noise-free, $20 \mathrm{~dB}, 10 \mathrm{~dB}$, and $0 \mathrm{~dB}$ conditions, respectively. Those corresponding improvements obtained by 1MaxCNN-E-MC are even better with $1.8 \%, 1.7 \%, 2.7 \%$, and $12.0 \%$. These lead to average absolute accuracy gains of $4.1 \%$ and $4.5 \%$ which are equivalent to relative error reduction rates of $69.5 \%$ and $76.3 \%$ for $\mathbf{1 M a x C N N - M C}$ and 1MaxCNN-EMC, respectively. Given the fact that multi-condition training was reported to result in little benefit on the task (for example, SIF-DNN-MC compared to SIF-DNN [1]), the performance of our multi-conditioned systems are quite impressive.

\subsection{Discussion}

Our proposed 1-max pooling $\mathrm{CNN}$ shows very promising performance even though we conservatively set the hyperparameters to very common values. Since there are many hyperparameters (e.g. the activation function, the filter width, the number of filters, the learning rate, the dropout rate, the regularization term $\lambda$ ), the chance to find a better set of values for them via parameter tuning is actually large. Furthermore, it is
Table 2: Classification accuracy (\%) comparison (results of the competitive systems courtesy of $[18,1,6])$.

\begin{tabular}{|l||clll|l|}
\hline System & clean & 20dB & 10dB & 0dB & mean \\
\hline \hline MFCC-HMM & 99.4 & 71.9 & 42.3 & 15.7 & 57.4 \\
MFCC-SVM & 98.5 & 28.1 & 7.0 & 2.7 & 34.1 \\
ETSI-AFE & 99.1 & 89.4 & 71.7 & 35.4 & 73.9 \\
\hline MPEG-7 & 97.9 & 25.4 & 8.5 & 2.8 & 33.6 \\
Gabor & $\mathbf{9 9 . 8}$ & 41.9 & 10.8 & 3.5 & 39.0 \\
GTCC & 99.5 & 46.6 & 13.4 & 3.8 & 40.8 \\
MP+MFCC & 99.4 & 78.4 & 45.4 & 10.5 & 58.4 \\
Dennis SIF & 91.1 & 91.1 & 90.7 & 80.0 & 88.5 \\
\hline SIF-DNN & 96.0 & 94.4 & 93.5 & 85.1 & 92.3 \\
SIF-DNN-MC & 94.7 & 95.8 & 92.1 & 87.7 & 92.6 \\
\hline SIF-CNN & 97.3 & 97.4 & 95.7 & 83.1 & 93.4 \\
SIF-IS-CNN & 97.3 & 97.3 & 96.2 & 85.5 & 94.1 \\
SIF-IS-DNN & 86.7 & 86.4 & 85.3 & 73.5 & 83.0 \\
MelFb-CNN & 97.7 & 97.5 & 94.7 & 70.3 & 90.0 \\
\hline 1MaxCNN & 98.0 & $\mathbf{9 8 . 1}$ & $\mathbf{9 7 . 3}$ & 75.5 & 92.2 \\
1MaxCNN-MC & 98.4 & $\mathbf{9 8 . 3}$ & $\mathbf{9 8 . 2}$ & $\mathbf{9 7 . 7}$ & $\mathbf{9 8 . 2}$ \\
1MaxCNN-E & 99.1 & 88.5 & 74.9 & 50.3 & 78.2 \\
1MaxCNN-E-MC & 99.1 & $\mathbf{9 9 . 0}$ & $\mathbf{9 8 . 9}$ & $\mathbf{9 7 . 5}$ & $\mathbf{9 8 . 6}$ \\
\hline
\end{tabular}

also worth further analyzing the sensitivity of the networks to these hyper-parameter values.

On the other hand, for simplicity we fixed the height of the filters equal to the number of frequency bins and only varied the width of the filters in time. And by this, we only conducted convolution in time direction. One possible improvement is to additionally allow convolution in frequency dimension, for example in different frequency subbands. However, the convolution should respect the order of the frequencies since it simply matters for audio signals. Lastly, it is also interesting to visualize the filters to see what the networks actually learn.

\section{Conclusions}

We presented a CNN network architecture that is efficient for robust audio event recognition. Compared to deep CNNs, our proposed architecture is relatively simple and more "shallow". Intuitively, with each convolutional filter coupled with 1-max pooling scheme, our CNNs based on the proposed architecture tend to extract the most discriminative and shift-invariant features from the audio signals for recognition. In addition, we can naturally deal with the temporal variations of audio events, thanks to the 1-max pooling scheme. In an evaluation on the standard task of robust audio event recognition, we obtain a relative error reduction of $76.3 \%$ compared to the reported results from the best deep CNN opponent.

\section{Acknowledgements}

This work was supported by the Graduate School for Computing in Medicine and Life Sciences funded by Germany's Excellence Initiative [DFG GSC 235/1]. 


\section{References}

[1] I. McLoughlin, H. Zhang, Z. Xie, Y. Song, and W. Xiao, "Robust sound event classification using deep neural networks," IEEE/ACM Trans. on Audio, Speech, and Language Processing, vol. 23, no. 3, pp. 540-552, 2015.

[2] O. Gencoglu, T. Virtanen, and H. Huttunen, "Recognition of acoustic events using deep neural networks," in $E U$ SIPCO 2014, 2014.

[3] G. E. Hinton, S. Osindero, and Y.-W. Teh, "A fast learning algorithm for deep belief nets," Neural Computation, vol. 18, no. 7, pp. 1527-1554, 2006.

[4] E. Cakir, T. Heittola, H. Huttunen, and T. Virtanen, "Polyphonic sound event detection using multi label deep neural networks," in Proc. 2015 International Joint Conference on Neural Networks (IJCNN), 2015, pp. 1-7.

[5] M. Espi, M. Fujimoto, K. Kinoshita, and T. Nakatani, "Exploiting spectro-temporal locality in deep learning based acoustic event detection," EURASIP Journal on Audio, Speech, and Music Processing, vol. 2015, no. 26, 2015.

[6] H. Zhang, I. McLoughlin, and Y. Song, "Robust sound event recognition using convolutional neural networks," in Proc. ICASSP, 2015, pp. 559-563.

[7] K. J. Piczak, "Envoronmental sound classification with convolutional neural networks," in Proc. 2015 IEEE Internationl Workshop on Machine Learning for Signal Processing (MLSP), 2015, pp. 1-6.

[8] L. Hertel, H. Phan, and A. Mertins, "Comparing time and frequency domain for audio event recognition using," arXiv:1603.05824, 2016

[9] R. Grosse, R. Raina, H. Kwong, and A. Y. Ng, "Shiftinvariant sparse coding for audio classification," in Proc. UAI, 2007.

[10] E. C. Smith and M. S. Lewicki, "Efficient auditory coding," Nature, vol. 439, no. 7079, pp. 978-982, 2006.

[11] M. R. DeWeese, M. Wehr, and A. M. Zador, "Binary spiking in auditory cortex," The Journal of Neuroscience, vol. 23 , no. 21 , pp. 7940-7949, 2003

[12] X. Glorot, A. Bordes, and Y. Bengio, "Deep sparse rectifier neural networks," in Proc. 14th International Conference on Artificial Intelligence and Statistics (AISTATS), 2011, pp. 315-323.

[13] Y. L. Boureau, J. Ponce, and Y. LeCun, "A theoretical analysis of feature pooling in visual recognition," in Proc. ICML, 2010, pp. 111-118.

[14] Y. Kim, "Convolutional neural networks for sentence classification," in Proc. EMNLP, 2014, pp. 1746-1751.

[15] A. Severyn and A. Moschitti, "Twitter sentiment analysis with deep convolutional neural networks," in Proc. SIGIR, 2015, pp. 959-962.

[16] N. Srivastava, G. Hinton, A. Krizhevsky, I. Sutskever, and R. Salakhutdinov, "Dropout: A simple way to prevent neural networks from overfitting," Journal of Machine Learning Research (JMLR), vol. 15, pp. 1929-1958, 2014.

[17] D. P. Kingma and J. L. Ba, "Adam: a method for stochastic optimization," in Proc. International Conference on Learning Representations (ICLR), 2015, pp. 1-13.
[18] J. Dennis, "Sound event recognition in unstructured environments using spectrogram image processing," Ph.D. dissertation, Nanyang Technological University, 2014.

[19] S. Nakamura, K. Hiyane, F. Asano, T. Yamada, and T. Endo, "Data collection in real acoustical environments for sound scene understanding and hands-free speech recognition," in Proc. EUROSPEECH, 1999, pp. 2255 2258.

[20] A. Varga and H. J. M. Steeneken, "Assessment for automatic speech recognition II: NOISEX-92: A database and an experiment to study the effect of additive noise on speech recognition systems," Speech Communication, vol. 12, no. 3, pp. 247-251, 1993.

[21] A. Sorin and T. Ramabadran, "Extended advanced front end algorithm description, version 1.1," ETSI STQ Aurora DSR Working Group, Tech. Rep., 2003.

[22] S. Chu, S. Narayanan, and C.-C. Kuo, "Environmental sound recognition with timefrequency audio features," IEEE Transactions on Audio, Speech, and Language Processing, vol. 17, no. 6, pp. 1142-1158, 2009. 